between the various forms of meningococci which have been shown by observers to give slight cultural and staining differences? Among the organisms tested in these observations it appears possible to differentiate three strains or variations of organisms-Belfast B. from Belfast M. and the present London organisms by the high opsonic power against young cultures as constantly demonstrated with normal serum; Belfast $M$. from Belfast $B$. by its very low opsonic power against young cultures as constantly demonstrated with normai serum; and Belfast $M$. from the present strain of London organisms by the normal index and absence of agglutination with the serum of Cases 6 and 7, the same serum giving a high index and marked agglutination reaction against the London organisms. It appears probable in order to obtain the fullest effects from a vaccine or serum treatment that the strain of organism employed in its preparation is of great importance.

I am greatly indebted to Dr. A. E. Garrod, Dr. A. F. Voelcker, Dr. W. S. Colman, and Dr. F. E. Batten for allowing me access to these cases and kindly permitting me to make use of their notes and treatment. Also to Dr. J. G Forbes for supplying me with subcultures of the meningococci isolated by him in these cases. My thanks are also due to Dr. T. Houston for allowing me to publish results with organisms and serum from Belfast.

Queen-street, W.C.

\section{AN INVESTIGATION OF SOME OF THE MORE UNUSUAL FORMS OF ACUTE CONJUNCTIVITIS.}

By A. C. HUDSON, M.D. Cantab., F.R.C.S. ENG., HOUSE SURGEON AT THE ROYAL LONDON OPHTHALMIC HOSPITAL; AND

P. N. PantoN, M.B., B.C. Cantab., M.R.C.S. Eng., ASSISTANT DIRECTOR OF THE LOUIS JENNER CLINICAL LABORATORY, ST. THOMAS'S HOSPITAL

THE bacteriological identification of the Koch-Weeks bacillus in 1885 and of the Morax-Axenfeld diplo-bacillus in 1896 as specific causes of conjunctivitis form important landmarks in the history of the study of this disease. The resulting types of inflammation are in most cases associated with such characteristic clinical features as to present a more or less diagnostic picture. In cases of doubt the diagnosis may be confirmed by an examination of films prepared from the discharge, the organisms having distinct morphological appearances and their cultural characteristics being rarely called for in making a diagnosis. IRecently accounts of these organisms have been published by Inglis Pollock $^{1}$ and by Usher and Fraser, ${ }^{2}$ whose papers dealing with the bacteriology of conjunctivitis are largely concerned with Koch-Weeks and diplo-bacillary infections and afford strong evidence that these organisms, together with the gonococcus, are responsible for the causation of the great majority of the more acute cases of the disease met with in this country. In any large clinic, however, a considerable percentage of cases of acute conjunctivitis is met with in which the clinical features would appear to present no such generally recognised rela. tion to their etiology. The aim of the present inquiry has been the investigation of cases of this nature met with by us during a period of 15 months, including several cases of gonorrhœal conjunctivitis, while cases of Koch-Weeks and diplo-bacillus conjunctivitis have been entirely, or almost entirely, omitted from this account, the cases encountered having been diagnosed either from clinical features or from film preparations, and no special media for the demonstration of these organisms having been employed.

TECHNIQUE.

In every instance two or more film preparations were made from the discharge and a culture was taken on to some form of solid medium, in most instances blood serum. One of the film preparations was stained by Gram's method and a second film was treated by Leishman's stain, whenever a cytological examination of the exudate was considered necessary. With the exception of some cases of gonorrhœal

1 Inglis Pollock : Tbe Bacteriology of Conjunctivis, Transactions of the Ophthalmological Society of the United Kingdom, vol. xxv., 1905. C. H. Usher and Henry Fraser: An Analysis of a Series of Con junctivitis Cases seen in Aberdeen. infection, in which an attempt at cultivation failed, no diagnosis was made from film preparations alone. In all, 105 cases were thus examined, but a certain small proportion of these include cases in which either the Koch-Weeks or Morax-Axenfeld bacillus was, in all probability, the main factor at work and are considered here only to complete the account of the other organisms present in addition. In a few cases films and cultures were taken also from other lesions in the body when these were thought to have any bearing upon the condition of the eyes. The cases were derived mainly from the Royal London Ophthalmic Hospital a considerably smaller number from the ophthalmic department of St. Thomas's Hospital are also included. The organisms met with were in order of frequency a diphtheroid bacillus (60 times), a white staphylococcus (28 times), the gonococcus (27 times), the staphylococcus aureus (24 times), a streptococcus (10 times), an intermediate staphylococcus (3 times), the diphtheria bacillus (twice), and an unclassified coccus (once).

The cases are grouped under the heading of the various organisms present, but since a mixed infection was frequent some overlapping is unavoidable.

\section{Group 1.-STREPTOCOCCI.}

There were altogether ten cases of streptococcal infection, and in each case an attempt was made to procure the full cultural characteristics of the organism in two instances, however, owing to the additional presence of a diphtheroid bacillus the streptococcus could not be isolated. In one other case the diphtheria bacillus, and in another both albus and aureus were present in addition; the former case is considered under Group 7. In the two cases in which the streptococcus was not isolated one was a severe attack with membrane formation, the other of moderate severity only and unaccom. panied by actual membrane. Five of the streptococci isolated were classed on the evidence of Gordon's tests as the streptococcus pyogenes according to the classification of Andrewes and Horder. ${ }^{3}$ In one of these five cases, a child, aged one week, a similar organism was present also in a sloughing hæmatoma of the face, and the fact that the mother died three days after delivery, presumably from some septic condition, points to the maternal passage as the probable source of infection. One of the pyogenes cases was accompanied by the diphtheria bacillus; there was wellmarked membrane formation in this case and corneal infiltration. In the remaining three cases membrane formation was present in each instance and in two cases the eye was destroyed. A different variety of streptococcus was isolated in two instances and should probably be classed with the streptococcus fæcalis group. The cultural characters were almost identical in the two cases. The coccus was Gram-positive and occurred in chains of from 10 to 20. On agar slant typical streptococcal colonies were found. On jelly slant, at $22^{\circ} \mathrm{C}$, no liquefaction. Litmus milk : acid, no clot. Gordon's media affected were : maltose, salicin, lactose, xylose, raffinose, arabinose, mannite, dextrose, and glycerine. Unaffected : erythrite, sorbit, and cane sugar. These media were identical in the two cases with the exception that litmus glycerine was acidified by the one organism and not by the other. In neutral red broth, anaerobically, a green fluorescence was produced by one organism only.

The occurrence of this same organism, of unusual characters, is of the more interest since the patients were brothers who attended the hospital at about the same time, and there is little doubt that they infected each other. Of these brothers one lost his eye ; the otber had only a mild attack of conjunctivitis which was associated with the onset of measles and which cleared up rapidly, affording a good example of the difference of personal resistance to the same organism. In another case, occurring in a small child with severe conjunctivitis accompanied by membrane formation, a streptococcus of unusual cultural characteristics was obtained. The organism was Gram-positive and occurred in very long chains. It formed very delicate colonies on agar and grew on jelly without liquefying it. It was transferred into a large number of Gordon's media and, with the exception of sorbit, acidified all of them. It produced green fluorescence in neutral red broth anaerobically. The organism was inoculated into the peritoneal cavity of a

3 F. W. Andrewes and J. T Horder: THE LANCET, Sept. 15th, 1906 p. 708 . 
mouse and cansed death in 12 hours; the streptococcus was recovered from the spleen, blood, and peritoneum.

The nine cases in which streptococci were considered the causative factor include some of the most severe cases met with in the series. In one case, however, in which the conjunctival inflammation preceded the onset of measles the symptoms were of a very mild character. The main clinical features are tabnlated below :-

Unilateral $\quad \ldots$\begin{tabular}{lllll|l} 
Ulceration of lid margins... & $\ldots$ & 2 \\
\hline
\end{tabular}

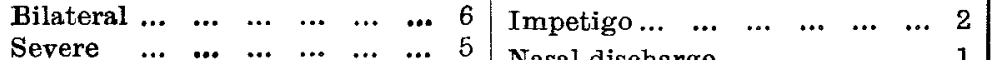

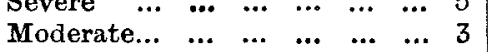

Mild ...

Fiar discharge

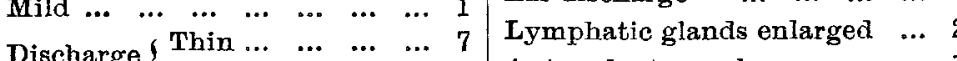

$\begin{array}{llll}\text { Membrane on conjunctiva } & \ldots & 5 & \text { Antecedent measles... } \\ \text { Highest temperature recorded }\end{array}$

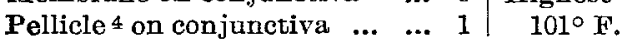

The following are clinical notes of illustrative cases :Cass 1.-A female, aged one year and two months. O.P., R.L.O.H.,
April 8th, 1907 (Mr. J. H. Parsons). Left eye inflamed four days, left lids red, but not much swollen. Palpebral and bulbar conjunctiva injected and slightly swollen. Very superficial membrane on palpebral conjunctiva of both lids, which could not be stripped off without some hæmorrhage. Thin muco-purulent discharge. Cornea normal. Left pre-auricular gland palpable. Right eye normal. later, the condition had considerably improved.

CASE 2. - A male, aged one year and three months. I.P. R.L.O.H. Jan. 1st, 1907 (Mr. J. H. Fisher). Right eye affected three days. Right lids cedematous, not brawny. Thick yellowish white membrane on conjunctival surface of both lids, which could not be removed without much bleeding. Some muco-purulent discharge. Right cornea very hazy. Left eye normal except for small patch of membrane on conjunctiva of left lower lid. No enlargement of lymphatic glands. Red spot on right cheek and forefinger. General condition fairly good. The disease rapidly progressed to a disastrous issue. Four days atter admission the entire right cornea had become infiltrated and staphylomatous, the membrane on the conjunctiva at this time stripping fairly easily the membrane on the conjunctiva at this time stripping fairly easily
and leaving bleeding spots. Left eye normal. Right eye subsequently ancised. Child left hospital one month after admission. Right socket was then clean except for small slough at the bottom, and urine conwas then clean except

CASE 3.-A male, aged two years and three months. I.P., R.L.O.H., CASE 3.-A male, aged two years and three months. I.P, , R.L.O.H.,
Jan. 10th, 1906 (Mr. J. B. Lawford). Whooping-cough eight months Jan. 10th, 1906 (Mr. J. B. Lawford). Whooping-cough eight months ago; delicate ever since. Bronchitis 14 days ago. Right lids swollen purulent discharge. Purulent mucocele. Deep haze of right cornea. purulent discharge. Purulent mucocele. Deep haze of right cornea.
Left eye normal. No enlargement of lymphatic glands. Affection of right cornea progressed rapidly, so that three weeks after admission the entire central portion had become yellow and staphylomatous. A fortnight later the eye was excised, the conjunctival inflammation having then almost subsided and there being no regurgitation from the having then almost subsided and there being

lacrimal sac. Convalescence uneventful. (Mr. Fisher). Brother of preceding patient. Very slight swelling of lids of both eyes with general conjunctival injection and a little watery discharge. Three days later it was reported that the patient had developed measles, but that the eyes were much better.

\section{Group 2.-STaphylococcus AuReus.}

This organism was present in 24 cases, in 19 of which it was either the only organism present or was associated with either a white staphylococcus or a diphtheroid bacillus ; in these cases it is classed as the causative organism of the disease. In the remaining five cases it was associated with the gonococcus twice, with the Klebs-Löffer bacillus, the Koch-Weeks bacillus, and a streptococcus, once each; in these cases it is considered as a secondary, though not necessarily a negligible, infection.

In view of the fact that the various staphylococci and other pathogenic organisms have on occasion been obtained from the normal conjunctiva, and that many of the attempts to produce by them experimental conjunctivitis in man and animals have failed, the majority of investigators into the bacteriology of conjunctivitis appear to regard the possibility of these organisms causing the disease with considerable reserve. Inglis Pollock ${ }^{\bar{x}}$ says, in agreement with Morax and Petit: "The pneumococcus and the Klebs-Löffler bacillus have occasionally, while the streptococcus and staphylococcus have as a rule, been found on the normal conjunctiva. Consequently the mere finding of these four organisms does not suffice for the diagnosis that a given case is due to them.

It is necessary to take into account the number of the microbes, their arrangements ...... etc." Similar views with regard to staphylococcal infection are expressed by Axenfeld ${ }^{6}$

"Bv the term " pellicle," used in this and subsequent tables, it is intended to describe a coherent sheet of fibrinous material which could be stripped off, leaving an injected but not bleeding conjunctival separated without hæmorrhage.

Loc. cit.

6 Theodor Axenfeld: Die Bakteriologie in der Augenheilkunde, 1907. and other writers ${ }^{7}$; yet the various staphylococci and even streptococci are found in the normal skin, and no one doubts that they may on occasion give rise to a subcutaneous abscess ; nor can a frequent failure to produce conjunctivitis be accepted as convincing, and, indeed, successful results have been recorded. In these cases no attempt has been made to estimate the number of microbes present, but their arrangement in the films was in each case noted. If the nature of the cells in the discharge plainly indicated an acute inflammation, if a culture taken from the fluid yielded an organism of known pathogenicity, and if similar organisms were present in the films (and preferably within the cells), that organism was considered the causative agent.

In 19 of the cases in this group, therefore, the causative agent was considered to be the staphylococcus aureus In three of these cases cultures were also taken from other lesions present in the body. In one case a pure culture of the staphylococcus aureus was obtained from the pus on incising an abscess of the neck; in this case, a small child, the conjunctival condition bad progressed to complete loss of the ejes and the child subsequently died from septic absorption. In one case a nasal discharge was present and from this a white staphylococcus and the micrococcus catarrhalis were obtained, but no aureus. In the third case an impetiginous condition of the scalp was present and aureus was obtained from one of the lesions; it is possible that the conjunctiva was secondarily infected from the head.

In the great majority of all the cases films from the discharge showed numerous microphages, many of which were greatly degenerated, the organisms appearing for the most part as Gram-positive diplococci, a certain number of these being within the phagocytes. It was exceptional to see a staphylococcal arrangement in the films and, if no cultural tests are made, both these and other organisms are liable to be mistaken for the pneumococcus. In every instance large, bright yellow colonies of a Gram-positive staphylococcus were obtained upon agar and in nearly all cases these were subcultered on to gelatin and produced liquefaction in from 24 to 48 bours. This early liquefaction of gelatin is considered by Axenfeld ${ }^{8}$ and others to be a good test of the relative pathogenicity of the various staphylococci.

In a few cases cultures were taken from the eye on two separate occasions, in each instance with identical results. As a control three strains, chosen indiscriminately, were passed through all the ordinary media and in addition through all Gordon's carbohydrate tests. The results were iound to differ in no way from those obtained with various strains of aureus derived from other parts of the body in disease. One of these strains was injected into the vein of a rabbit; the animal died in ten hours ; no lesions were found post mortem and a pure culture of a white staphylococcus was obtained from its spleen. A further inoculation of the original culture was made into the hind leg of a guinea-pig and resulted in a local abscess; the animal was killed one week later and cultures were taken from the spleen and the abscess ; in both cases a white staphylococcus only was obtained. This coccus on being submitted to Gordon's tests gave results absolutely identical with those of the original yellow coccus. The result is of interest as indicating the possibility of an aureus losing its power of pigment production and appearing as an albus, though still retaining its other cultural characteristics.

The 19 cases in which the staphylococcus aureus was believed to be the causative organism were on the whole of severe type; only four conld be designated as mild. The leading features of the 18 cases of which clinical notes are available are presented in the following table :-

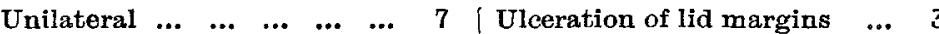

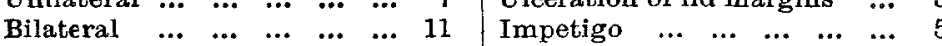

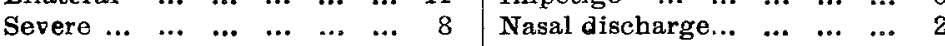
\begin{tabular}{lllllll|lllll} 
Moderate & $\ldots$ & $\ldots$ & $\ldots$ & $\ldots$ & $\ldots$ & 6 & Ear discharge & $\ldots$ & $\ldots$ & $\ldots$ & $\ldots$
\end{tabular} \begin{tabular}{llllllll|lll} 
Mild & $\ldots$ & $\ldots$ & $\ldots$ & $\ldots$ & $\ldots$ & $\ldots$ & 4 & Lymphatic glands enlarged $\ldots$ & 8
\end{tabular}

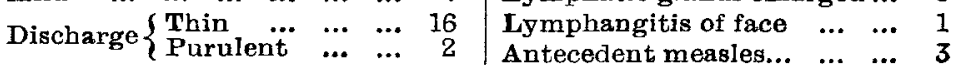

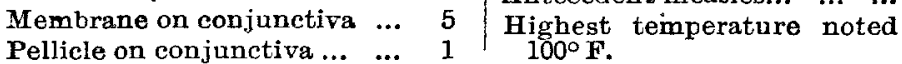

The following are brief notes of illustrative cases:-

CA8E 5.-A female, aged 27 years. O.P., R.L.O.H., Oct. 8th, 1906 (Mr. A. Lawson). Both eyes inflamed 24 hours. Slight injection and very slight swelling of conjunctiva of both eyes. A very little watery discharge. Cornex normal. Both preaui icular 'glands just palpable but

? J. H. Parsons: The Pathology of [the Ey e, vol. i., part 1. 
not tender. Four days later the patient was quite well, except for slight stickiness of lids after sleep.

CASE 6.-A male, aged 16 years. I.P., R.L.O.H., April 3rd, 1906 (Mr. W. T. H. Spicer). Right eye watery and painful three days; running from nose and sore throat for same period. Right lids very slightly swollen with congestive discolouration. Conjunctiva of both lids covered leaving an injected, but not swollen, conjunctival surface. Bulbar conjunctiva considerably injected but not swollen; pellicle did not extend to it. Watery discharge. Cornea not affected. Infection of skin of both lid margins with linear distribution on upper, in circular patches nn lower, lid ; large angular area at outer canthus. Infective process had proceeded to varying extent in different areas, so that it could be seen to be initiated by a subcuticular infiltration covered by a shiny cuticle which later lost its lustre, giving rise eventually to an opaque, greyish membrane. Right preauricular gland enlarged, hard, and tender; cervical glands on both sides of neck palpable. Red, slightly papular, eruption on right side of face. Left conjunctiva and lids normal. No membrane on fauces. Two days later left conjunctiva became slightly injected and eye watery. A week later, when the patient was dis char

CAse 7.-A female, aged seren years, I.P., S.T.H., July 20th, 1906 (Mr. Lawford). Left eye affected five days. History of exposure to diphtheritic infection. Child pale and fretful. Left eye : lids swollen, closed, and somewhat brawny. Palpebral conjunctiva covered by closed, and somewhat brawny. Palpebral conjunctiva covered by thin, yellowish pellicle which extended to both fornices and slightly on to the bulbar conjunctiva, and on separation left a bleeding surface slight chemosis and congestion of bulbar conjunctiva. Muco-purulent discharge. Cornea bright. Left preauricular gland enlarged. Right eye normal 6000 units of diphtheritic antitoxin were given shortly after admission to hospital. Highest temperature during stay in
hospital $100^{\circ} \mathrm{F}$. Patient was discharged in 11 days, when the swelling and membrane had nearly disappeared.

CASE 8.-A female, aged seven years. I P., R.L.O.H., Sept. 29th, 1906 (Mr. R. M. Gunn). Yellow sty on left lower lid noticed four days ago. Left eye closed, cheek swollen, and preauricular gland enlarged two days. Discharge from nose noticed 24 hours. Left eye : greyish yellow absolutely adherent membrane involved almost entire margin of upper and outer half of margin of lower lid : great induration of lids ; palpebral conjunctiva not seen, bulbar conjunctiva hyperæmic. Some thin discharge. Right eye : similar membrane on outer part of upper lid margin; did not extend on to conjunctiva, which was normal. Cornex normal. Lymphangitis of left side of face and tender swelling of right and left preauricular and cervical glands. Membrane inside left nostril. Fauces normal. Knee-jerks present. Three days later small circular patch of membrane in skin of face just below right lower lid. A week after admission the condition had greatly improved, but there was a iittle thin greyish membrane in the conjunctiva of the left lower lid continuous with that on the cutaneous surface; conjunctiva eisewhere simply thickened and injected; bead of yellow discharge at inner canthus. A week later patient was discharged cured.

In the following case the Koch.Weeks bacillus was found associated with staphylococcus aureus.

CASE 9-A female, aged 21 years. O.P., R.L.O.H., Feb. 14th, 1906 (Mr. E. Treacher Collins). Left eye excised three years ago. Sore throat and swelling of glands in left side of neck four days. Swelling of lids and discharge from left conjunctiva three days. The conjunctiva of the left socket presented a shaggy yellow appearance owing to a superficial necrosis of the conjunctiva of the lids ; a similar process had involved the lid margins around the bases of many of the lashes. Much flaky yellow discharge. Some cdema of left side of face; left preauricular and cervical $1 \mathrm{ymphatic}$ glands considerably enlarged and slightly tender. Right eye normal; slight cdema of right side of face. Hyperæmia of fauces; no membrane. Temperature $99.8^{\circ} \mathrm{F}$. Patient looked and felt fairly well. Four days later the conjunctiva of the socket was almost normal in appearance.

In this case the clinical features were so much at variance with those characteristic of a simple Koch.Weeks infection as strongly to suggest that it was the staphylococcus aureus which had played the chief rôle in the production of the clinical picture.

\section{Group 3. - AN InTermediate Staphylococcus.}

This organism was present tbree times, twice in pure culture and once in association with a diphtheroid bacillus; in each case it was considered the cause of the lesion. In each instance cultures of a Gram-positive staphylococcus were obtained, which, after prolonged exposure to sunlight, remained of a colour intermediate between aureus and albus. In one case a subculture upon gelatin showed commencing liquefaction in 48 hours, becoming complete in six days. In the remaining two cases the fullest cultural characters were taken and were found to be similar to those obtained from the aureus strains of the preceding group. The three cases occurred in children under six years of age. The first case was a rather acute, and extremely intractable, bilateral conjunctivitis. There was a watery discharge with a few flakes, slight thickening of the lid margins, and some enlargement of both preauricular lymphatic glands. The second case was unilateral and associated with vesicle formation on the lid margins, followed by superficial necrosis which extended to the palpebral conjunctiva. The preauricular gland was enlarged and the temperature reached $101^{\circ} \mathrm{F}$. The third case was a mild bilateral conjunctivitis with superficial membrane formation in both lower fornices.

Group 4.-AN UNGLAssified Coccus.

This was present in one instance and was associated with a diphtheroid bacillus. The films showed numerous micro. phages and a number of Gram-positive diplococci, some of which were intracellular. On agar small, round, white colonies were obtained which were intermediate in size between those usual for a streptococcus and those for a staphylococcus ; they consisted of Gram-positive cocci in pairs and small clumps; in broth they appeared in small clumps and in short chains of two and four. In neutral red broth they grew well anaerobically but without changing the colour of the medium. Gelatin was liquefied in eight days. Milk and lead acetate were unaltered. No indol was formed in peptone broth. Of the carbohydrate media, lactose, maltose, cane sugar, and arabinose were acidified ; xylose, mannite, salicin, raffinose, erytbrite, and sorbit were unaltered. The coccus was non-pathogenic to a guinea-pig. We have isolated an exactly identical organism from a case of acute cellulitis. The organism appears to have some characters common to both the streptococci and staphylococci. The case from which it was derived in this instance was a unilateral conjunctivitis without corneal lesion in a child five years of age. There was considerable swelling of the conjunctiva with thin mucinoid discharge containing a few thicker flakes. The preauricular lymphatic gland on the same side as the affected eye was just palpable. The patient did not attend the hospital a second time.

\section{Group 5.-STAPHYLococcus ALBUS.}

This organism occurred altogether 28 times. On 12 occasions it was associated with other pathogenic organismsnamely, aureus five times, gonococcus three times, KochWeeks bacillus twice, Morax-Axenfeld bacillus, and a streptococcus once. In the remaining 16 cases it was either the only organism present or was associated with a diphtheroid bacillus. These 16 cases were accompanied by a considerable conjunctival discharge, the cells in which were mainly polynuclear; in the great majority of the films Gram-positive cocci were present, and many of these were inside the lencocytes. Large white colonies were obtained on agar in each case and these remained white on being exposed to the light for some days. In the majority of cases a subculture was taken on to gelatin and this was, as a rule, liquefied in from two to (more commonly) seven days. In three cases cultures were taken also from a nasal discharge which was present; on one occasion albus only was grown from the nose, once albus and the micrococcus catarrhalis, and once albus and aureus. In the last case the complete cultural characters of the albus, from the eye and from the nose, were investigated. The results were strictly identical in two cases.

Since a white staphylococcus is stated to have been frequently isolated from the normal eye, and since this class of organism is frequently one of low virulence, the question has arisen as to whether it can ever give rise to an acute conjunctival infection. In 16 out of these 28 cases this was the only organism of known pathogenicity found; it was present not only in the cultures but as a rule in the films and frequently within the phagocytes; moreover, in three cases it was present also in another, and possibly the primary, lesion. If albus is not allowed to be the cause of the infection it must be supposed either that a known causative agent was overlooked, or that some unknown organism was the cause of the disease. Every precaution was taken to guard against the former contingency and the latter supposition seems unnecessary, for some species of albus approach closely to the virulent staphylococcus aureus in their cultural characters, and there is a wide range both in the virulence of any particular organism and the resistance of any particular person; there is, too, sufficient evidence, both clinical and experimental, that a white staphylococcus can produce an acute inflammation. The cases in which a staphylococcus albus appeared to be the causative organism were almost all of mild type. In one case, however, a wasted unhealtby child, the infection was of a very severe character.

The main clinical features are presented in the following table :-

Unilateral

Bilateral.

Severe

Moderate

Mild

Discharge $\left\{\begin{array}{l}\text { Thin } \\ \text { Purul }\end{array}\right.$

Membra

Pellicle
Ulceration of, or membrane on, lid margins Impetigo

Nasal discharge

Lymphatic glands enlarged ... Lymphangitis of face

Antecedent measles ...

Highest recorded temperature $99 \times 4 \circ \mathrm{F}$. 
The following are notes of illustrative cases:-

Case 10.-A female, aged 19 days. O.P., R.L.O.H., April 6th, 1906 (Mr. P. Flemming). Discharge from eves noticed third day after birth. Some swelling of lids of both eyes. Palpebral conjunctiva succulent and purplish. Muco-purulent discharge. Corneæ normal. Eyes quite well in five days.

CASE 11.-A female, aged 30 years. O.P., R.L.O.H., Sept. 24th, 1906 (Mr. W. Lang). Right eye affected 36 hours. Pink œedema of both right lids, extending inwards to nose and outwards on to cheek. Palpebral and bulbar conjunctiva considerably swollen; slight follicular enlargement. Thin discharge. Cornea normal. Right preauricular and cervical lymphatic glands enlarged. Left eye not affected. and cervical lymphatic glands enlarged. Ceft eye not affected. days after the first attendance the left conjunctiva became injected and days after the first attendance the left conjunctiva became injected and
slightly swollen without enlargement of lymphatic glancs. Both eyes slightly swollen without enlargeme
were almost well four days later.

CASE 12. -A male, aged 13 years, O P., R.L.O.H., Oct. 24th, 1906 (Mr. Lawford). Impetigo of left foot "on and off for a long time." (Mr. Lawford). Impetigo of left foot "on and off for a long time." Right nostril sore for three or four months. Right eye affected four days. Right lids swollen and indurated; many small fattened vesicles on margins of both lids. Palpebral conjunctiva very red, swollen, and follicular. Discharge thin with a few flakes. Cornea normal. Right preauricular and submaxillary lymphatic glands much swollen.

Temperature $99^{\circ} \mathrm{F}$. Rapid improvement under treatment.

CAST 13.-A female, aged one year, O.P., R.L.O.II., Feb. 14th, 1906 (Mr. Gunn). Discharge from right eye five days. Ulceration of lower lid noticed one day. Slight swelling of right lids ; some ulceration of skin at inner canthus. Palpebral and bulbar conjunctiva slightly swollen; superficial yellowish grey necrosis of conjunctiva of both lids. A very little thin discharge. Cornea normal. Left eye not affected. A week later the condition of the conjunctiva had greatly improved, but a minute corneal ulcer had developed. This had healed 17 days later.

The line of treatment adopted in the staphylococcal and streptococcal infections was as follows. In those cases in which the conjunctival inflammation was not associated with membrane formation or ulceration of the lids the conjunctiva was brushed over daily with a solution of silver nitrate, usually of the strength of five grains to the ounce and the conjunctival sac was washed out every four to six hours with some antiseptic lotion: perchloride of mercury in the strength of one part in 10,000 appeared to be the most satisfactory for this purpose. Where membrane formation was present a 20 per cent. solution of argyrol was nsed instead of nitrate of silver, and was found to have no tendency to produce extension of the necrotic process, such as has been observed in cases of this type after the use of silver nitrate. In those cases in which there was an associated infection of the skin of the lids or their margins without corneal lesions the application of boric fomentations to the lids, by bringing about a rapid restoration of the skin to a healthy condition, was found to act as a valuable adjuvant to the treatment of the conjunctival sac. Corneal ulceration appeared to afford a contraindication to the use of fomentations. The frequent application of moist heat to the lids in the form of hot bathing was found to have a beneficial effect in cases with this complication. In the more severe cases the general condition of the patient was frequently a factor demanding special attention. In a few cases a tardy convalescence was associated with a residual infection of the lash follicles, evidenced by thickening of the lid margins and hypersensitiveness when the cilia were stroked with the finger; satisfactory progress was usually maintained by daily epilation of loose lashes and scrubbing of the lid margins with a pledget of wool soaked in a 15 per cent. solution of protargol.

A percentage comparison of the main features met with in the cases associated with the streptococci, staphyloccccus aureus, and staphylococcus albus gives the following results :-

\begin{tabular}{|c|c|c|c|c|c|c|c|c|c|c|c|}
\hline- & 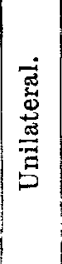 & 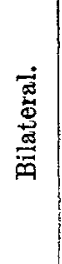 & 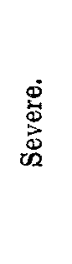 & 害 & 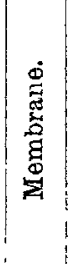 & 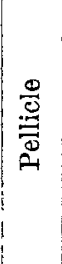 & 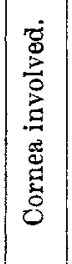 & 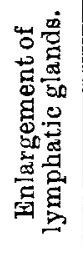 & 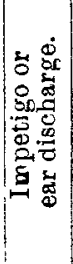 & 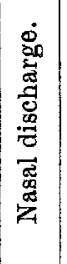 & 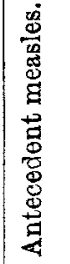 \\
\hline & $\%$ & $\%$ & $\%$ & $\%$ & $\%$ & $\%$ & $\%$ & $\%$ & $\%$ & $\%$ & \\
\hline Streptococci ... & $33 \cdot 3$ & $66 \cdot 7$ & $55 \cdot 5$ & $9 \cdot 0$ & $55 \cdot 5$ & $11 \cdot 0$ & $33 \cdot 3$ & 222 & $33 \cdot 3$ & $11 \cdot 0$ & \\
\hline $\begin{array}{c}\text { Staphylococcus } \\
\text { aureus } \\
. .\end{array}$ & 377 & $62: 3$ & $44 \cdot 4$ & 228 & $27 \cdot 7$ & 222 & $33 \cdot 3$ & $44 \cdot 4$ & $37 \cdot 7$ & $11 \cdot 0$ & \\
\hline $\begin{array}{l}\text { Staphylococcus } \\
\text { albus ... ... }\end{array}$ & 31.0 & $69 \cdot 0$ & 625 & $87 \cdot 5$ & 25 & $12 \cdot 5$ & 187 & $31 \cdot 25$ & $37 \cdot 5$ & 250 & \\
\hline
\end{tabular}

The great majority of these infections occurred in young children. In all the groups a thisk purulent discharge was rarely met with, the character of the discharge being usually glairy with some solid flakes, or watery. Conjunctival hæmorrhages, so frequently met with in Koch-Weeks conjunctivitis, were observed in only two of the cases, both albus infections; an associated "angular" affection of the lid margins was not met with. A possible exposure to con tagion was noted in one of the anreus and in one of the albus cases, and in the mild streptococcal affection occurring during the prodromal stage of measles.

From the above considerations it is evident that the clinical symptoms in the various infections presented many points of similarity, but that the severity of the type of infection showed a direct relation to the generally recognised pathogenicity of the causative type of organism. Membrane formation was met with in association with all the organisms in the group. The phenomenon of membranous formations was particularly observed, and was met with in various forms. In some of these a mere fibrinous pellicle was present adhering loosely to a conjunctival surface which presented when exposed an injected or granular appearance. In other cases the inflammatory process had apparently pro ceeded further, resulting in the destruction of the most superficial layers of the conjunctiva, which had become incorporated in the membranous structure; such membranes were more firmly adherent, and their removal was associated with conjunctival hæmorrhage. In other c»ses, again, the process had extended still more deeply, resulting in a more or less extensive necrosis of the tissues; such membranes were firmly adherent so that their removal would have entailed the display of an ulcerated surface.

The question of the relative frequency of glandular enlargement in the various infections presents a point of interest; such enlargement was usually observed in the severe cases of staphylococcal conjunctivitis, but was notably absent in the most virulent streptococcal cases.

Corneal ulceration, although met with in all the types of infection, occurred in its severest and most intractable form in the streptococcal infections; indeed, there can be no doubt that the supervention of this complication in such infections renders the prognosis as to the preservation of the affected eye exceedingly grave, while the spread of the infection to the interior of the globe subsequent to perforation is liable to prove a menace to the actual life of the patient.

\section{Group 6. -THe Gonococcus.}

This organism was obtained altogether 27 times ; but this number cannot be taken as the proper proportion of its occurrence in the series, since many cases of ophthalmia of the newly born which presented themselves were not investigated. The majority of cases of gonorrhoeal infection of the eyes in older children or adults are included. In 13 cases the gonococcus was associated with a diphtheroid bacillus. A white staphylococcus was present in two of these cases and in one other case, or in three cases altogether. The staphylococcus aureus was present twice, while the gonococcus was the only organism found in 11 cases. In only about one-fourth of the cases was a growth of the gonococcus obtained, the bacteriological diagnosis of the remainder resting with the examination of the films. No clinical difference in the severity of the case could be made out between those patients from whom a growth of the organism was obtained and in those from whom none was obtained; nor did the presence or absence of a diphtheroid bacillus appear to affect the clinical course. The two cases associated with aurens were both severe, in on case the affected eye being lost, while the other case was complicated by corneal ulceration. Of the three cases accompanied by albus two were of moderate severity and one was a mild affection. In five of the cases in older patient films were examined from the associated urethral or vaginal discharge and gonococci were found in each instance.

The question of the presence of coarsely granular eosinophile cells in the discharge and in the blood was particularly investigated. On the whole a slight but fairly constant increase in the eosinophile cells of the blood and of the pus was found to be present in the gonorrbcal infections; it was often, however, inconspicuous and occasionally absent, so that no reliance can be placed upon it in diagnosis. The presence of eosinophiles in the blood in the proportion of 2 or more per cent. was considered to constitute an eosinophilia.

${ }^{9}$ Cf. Jessop: Transactions of the Ophthalmological Society of the United Kingdom, vol. xxii. 
The question of the existence of a gonorrhoal eosinophilia is considered by Cabot,,$^{10}$ who considers an eosinophilia of 4.2 per cent. as within the normal limits, a number with which it is impossible to agree. In these cases no relation could be made out between the severity of the infection and the number of eosinophile cells present in either the discharge or the blood. Of these 27 cases 18 occurred in children under one month old and nine in older children or adults. The actual number of children under one month otd examined in this series was 22 . In 18 of these gonococci were present; in 4 they were found neither in the films after a prolonged search nor in the cultures. The organisms present in these four cases were a streptococcus twice, a white staphylococcus once, and a diphtheroid bacillus once.

\section{Group 7.-The Diphtheria Bacilius.}

In 105 cases examined this organism was met with twice only, and since these cases represent only a small proportion of the total number of patients reviewed, the diphtheria bacillus would seem to be an unusual cause of conjanctivitis. In both these cases another organism was present-namely, in one instance the staphylococcus aureus and in the other the streptococcus pyogenes. Both patients were children suffering from a moderately severe type of conjunctivitis which was, however, not clinically considered to be diph theritic. A nasal discharge, from which the diphtheria bacillus was cultivated, was present in both cases and was probably the primary source of infection.

It is well known that diphtheroid bacilli are frequently present in the conjunctival sac, as also in the nasal mucous membrane, and that these organisms cannot be distinguished from the genuine Klebs-Löffler bacilli either morphologically or by their cultural appearances upon solid media. The most useful medium for distinguishing the two organisms is undoubtedly litmus dextrose broth, which is acidified by the diphtheria bacillus in 12 hours at $37^{\circ} \mathrm{O}$. The diphtheroid bacilli met with in the eye affect dextrose so rarely that if a bacillus is present in the discharge and is found to acidify litmus dextrose in from 12 to 24 hours it is advisable to administer antidiphtheritic serum, whether the case clinically resembles diphtheritic conjanctivitis or not. By taking the initial culture on to a solid medium and by subculturing from that into dextrose a bacteriological diagnosis sufficien for practical purposes can if necessary be made in 24 hours. Since, however, diphtheroid bacilli are exceptionally met with which are capable of acidifying dextrose the diagnosis can be completed only by animal inoculation. In these cases two cubic centimetres of a thin suspension of the bacill were injected subcutaneously into guinea-pigs, resulting in both instances in the death of the animal in from 30 to 36 hours with the ordinary post-mortem appearances of the disease; in one case the bacillus was recovered from the spleen.

The following are clinical notes of the two cases in which the Klebs-Löffler bacillus was found :-

CASE 14.-A male, aged one year and 11 months, I.P., S.T.H." Feb. 15th, 1907 (Mr. Lawford). Child ailing one week : croupy cough, no history of exposure to diphtheria. Right eye : both lids swollen muco-purulent discharge; thin vellow membrane on conjunctiva of both lids, which was peeled off with difficulty, leaving a bleeding surface. Centre of cornea infiltrated. (When first seen, five days before admission, the membrane was limited to the lower lid, extending to the upper lid two days later; the cornea remained clear until the day before admission.) Left eye normal. Profuse nasal discharge and excoriation of left nostril. No membrane on tonsils. Temperature normal. Urine contained a strong trace of albumin. Klebs-Löffler bacillus and strepto coocus pyogenes present in the conjunctival sac. On the first day after units of dipo units, and on the third, fourth, and fifth days 4000 ing an antitoxin rash two days after the last in was limited to the use of antiseptic lotions, the use of nitrate of silver, which had been applied before admission to hospital, being discarded. The lids remained free frefore admission to hospital, being discarded. valescence, although protracted, was satisfar the fourth day and conadmission a pustule was noted on the right cheek below the right ese. admiston a poted on the right cheek below the right e ce. Cultures taken at about this date showed streptococci but no bacill. The temperature on several occasions was as high as $100^{\circ} \mathrm{F}$. but never rose above this, and the urine was free from albumin three weeks after admission. The patient left hospital on March 25th, the conjurctiva being then free from signs of infammation and the cornea having healed. He was readmitted on May 13th, the right eye having bee inflat

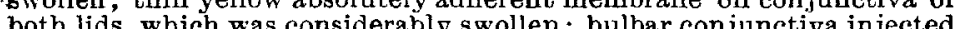
but very slightly swollen Purulent discharge Cornea not affected. ut Left e narmal. Right preauricular and submaxillary lymphatio The bacilli were found either in culture or in film preparations four days

10 Cabot: Clinical Examination of the Blood, 1904 later, under treatment with perchloride of mercury lotion, the con dition showed considerable improvement, the membrane formation conjunctiva.

We are much indebted to Mr. Lawford for his permission to publish this case.

The second case was of milder type with superficial membrane on the conjunctiva of both lower lids, enlarged preauricular glands, and thin nasal discharge. The fauces were normal. The patient recovered in 12 days without the use of antitoxin. There were no corneal ulceration, no albuminuria, and no subsequent loss of knee-jerks.

\section{Group 8.-Diphtheroid BachluUs.}

In 60 , or rather more than half of the cases examined, bacilli which morphologically resembled the diphtheria bacillus were present, either in the films, the cultures, or in both. In 49 of the cases other organisms were present in addition; in none of these cases was there any reason to suppose that the diphtheroid bacilli were the causative agents. In the remaining 11 cases, however, these bacilli were the only organisms discovered.

The term "diphtheroid bacillus" jncludes a variety of organisms, and a number of these have been investigated by Graham-Smith ${ }^{11}$ and others with a view to their differentiation. The majority of those met with in the eye in this series, however, appeared to conform to the same type, while a few presented some cultural modifications; those which we have investigated from other parts of the body grew more abundantly in broth and more readily produced acid reaction in litmus dextrose broth. The name "diphtheroid bacillus" does not, in this account, include Hoffmann's bacillus, which is morphologically distinct from the Klebs-Löffler bacillus, and which was not met with in these cases. The frequent presence of these bacilli, both in the healthy and in the diseased eye, would seem to indicate for them a mainly saprophytic existence ; they may frequently be seen in large numbers in a film within the epithelial cells and unaccompanied by evidence of inflammation.

The diphtheroid bacilli met with showed the following morphological and cultural characters. On agar a good growth, forming in from 12 to 24 hours small pin-point, rather opaque, colonies with slightly irregular margin and presenting a paler periphery with a ditch-like interval between centre and periphery. Films from these colonies show beaded bacilli with the typical arrangement of the diphtheria bacillus, large numbers having well-marked club-shaped ends. In films taken from the water of condensation masses of very beaded bacilli are present, many of them arranged in tight clumps, while streptococcal forms in chains of about eight to ten are not infrequent. The bacilli stain deeply by Gram's method, but their characters are best shown by Löffler's methylene blue ; one culture which was kept for three months was found to have lost its property of retaining the stain by Gram's method. On blood agar and nasgar similar colonies are obtained. The bacillus grows well on gelatine at $22^{\circ} \mathrm{C}$. ; there is no liquefaction of the medium. In broth and in the various carbohydrate media no growth as a rule takes place, but the organisms maintain their existence. In litmus milk growth is often absent or is slight.

In the 11 cases in which diphtheroid bacilli alone were found the causative organism may have been overlooked, but in a few of these the features were clinically anomalous and more than one examination was made with identical results. In a high percentage of these cases the bacilli showed some cultural variations to the type usually observed and it is possible that some species of the diphtheroid bacilli may possess properties pathogenic to man. In a few instances animal inoculations were made but with negative results; the injections were in each case made subcutaneously into the legs of guinea-pigs.

\section{CONCLUSIONS.}

That both staphylococcus albus and staphylococcus aureus, as well as streptococci, are capable of exciting an acute conjunctival inflammation. That these organisms when they are present as the only pathogenic bacteria in an inflammatory conjunctival discharge should be considered as the causative agents. That these organisms give rise to a considerable percentage of all cases of acute conjunctivitis. That the severity of the type of inflammation they produce varies

G. S. Graham-Smith: The Action of Diphtheria and Diphtherialike Bacilli on Yarious $S$ ugars and Carbohydrates, Journal of Fygiene July, 1906. 
on the whole directly with the generally recognised pathogenicity of the members of the group, the most severe cases being associated with the streptococci and the least severe with the white staphylococci; but that the severity of the symptoms produced by any of the individual organisms may vary considerably with the idiosyncrasies of the patient. That there is reason to suppose that these bacteria, when associated with other pathogenic organisms, tend to increase the severity of the conjunctival inflammation. That the types of acute conjunctivitis due to these organisms, although presenting no pathognomonic features, are not infrequently suggestive of their etiology. That staphylococcal, as well as streptococcal, infection of the conjunctiva is liable to be associated with membrane formation. That conjunctivitis due to the diphtheria bacillus is uncommon and may be difficult to diagnose on clinical grounds. That diphtheroid bacilli are present in a high percentage of cases without appearing to influence the nature of the processes. It is probable that they represent more than one species and it is possible that some of these may be pathogenic to man; but there is no evidence that they may ever become transformed into the genuine diphtheria bacillus. That sporadic cases of pneumococcal conjunctivitis must be of rare occurrence in London, not a single case being met with in this series. Inasmuch as the diagnosis of this organism from a streptococcus may be a matter of great difficulty, its identification by film preparations only or by incomplete cultural investigations cannot be relied on ; the mere appearance of encapsulation of the organisms in the films is most unreliable as a means of diagnosis. These observations agree with the investigations of others as to the rarity of organisms of the bacillus coli group as causes of acute conjunctivitis.

In conclusion, we have to thank the members of the staff of the Royal London Ophthalmic Hospital and of the Ophthalmic Department of St. Thomas's Hospital for permission to make use of the material from their cases. All the inoculation experiments were performed by Mr. L. S. Dudgeon at St. Thomas's Hospital and some of the organisms isolated were submitted to Gordon's tests by him. We are also indebted to him for his advice on many occasions. We have also to thank Mr. H. E. Gotelee, Mr. W. C. A. Ward, and other ophthalmic house surgeons at St. Thomas's Hospital for their assistance in obtaining the clinical notes of some of the cases.

\section{Clinital 侽otes:}

\section{MEDICAL, SURGICAL, OBSTETRICAL, AND THERAPEUTICAL.}

NOTES OF A CASE OF DISTAL LIGATURE OF THE COMMON OAROTID ARTERY FOR ANEURYSM.

By J. Crawford Renton, M.D. Edin.,

SURGEON AND LECTURER OY CLINICAL SURGERY, WESTERN INFIRMARY, GLASGOW; EXAMINER IN SURGERY AND CLINICAL SURGERY IN THE UNIVERSITY OF ABERDEEN.

ThE patient, a female, aged 47 years, was sent to see me by Dr. J. Holms of Paisley in October, 1906. She was suffering from a pulsating swelling immediately above the right sterno-clavicular articulation and she complained of the throbbing and of a pain in the region of her heart giving rise from time to time to palpitation. On examination the tumour and its pulsation were distinctly visible and on palpation both were easily felt; the two radial pulses were equal in strength and character. Considering the above facts it was evident that she was suffering from an aneurysm of the first part of the right common carotid artery not involving the innominate or subclavian. I therefore advised that a distal ligature should be applied to the common carotid. Professor R. Stockman saw her in consultation and was satisfied that her general arterial condition was otherwise healthy. The operation was performed, a silk ligature being carried round the artery above the omohyoid. The wound healed by first intention; no headache was complained of, and the patient at the end of four weeks was allowed to lie on the sofa. On examination at the end of this time no pulsation was visible in the swelling and only moderate beating was noticed on palpation. Three months after the operation the patient was unfortunately in a tramcar collision, after which the pulsation increased for some weeks. This has now to a large extent subsided and the patient expresses herself as free from any discomfort.

Remarks.-Ever since Brasdor suggested and Wardrop carried out distal ligature of the common carotid in 1825 cases of aneurysm to which this method is suitable have occurred from time to time, but they are sufficiently rare to allow of the above case being recorded. The great improvement in the aneurysmal condition and the increased comfort which the patient now enjoys seem to justify the operation.

Four weeks after this case was under care Dr. Holms asked me to see another patient with an aneurysm exactly similar to the above. She was 74 years of age and had general arterial disease so that I did not advise operation. It is curious to have had the opportunity of seeing two such cases within six weeks, the more so that no parallel case has come before us for consideration for several years.

Glasgow.

\section{A CASE OF FOREIGN BODY IN THE FEMALE} BLADDER.

BY WiLlIAM HooK, L.S.A.

THE patient in the following case was a woman, aged 22 years, who was brought by her mother to my surgery on June 15th. I was informed that owing to difficulty in leaving her work for lavatory purposes her daughter had inserted a cork into her "front passage." An examination of the vagina showed that there was no foreign body there, so I assured her that it must have been expelled and as she no longer felt pain she went home. Returning, however, very shortly she said that she was sure the cork was still there. I then made an inspection, and finding the urethra very low and dilated without the slightest difficulty I passed my finger into the bladder and there came on the cork. About an hour later, having obtained the assistance of $\mathrm{my}$ friend, Mr. R. J. Heatly, of Stockwell-road, who put the girl under an anæsthetic, I made an attempt at removal but was unable to locate the foreign body. My friend, however, who has a long forefinger, quickly did so and after failing to grasp it with suitable forceps ultimately succeeded in manipulating it through the urethra. I saw the girl two days later and she then appeared none the worse for her foolish act. The cork measured an inch in length and half an inch in diameter.

Clapham, S.W.

\section{A CASE OF PROLONGED RETENTION OF FOOD IN} AN CESOPHAGEAL POUCH.

By William AYres, M.D. DUB.

THE patient, who was a female, 38 years of age, suffering from pulmonary tuberculosis, sent for me as she was troubled with a severe cough. As there was a large cavity opposite the third and fourth ribs on the left side and as there was a large quantity of secretion this cough was naturally thought to be one of the ordinary concomitants of the condition present. A few days subsequent to my first attendance after a violent paroxysm of coughing a peculiar-looking yellow mass was expectorated, The cough was much relieved in a few days. The pathological report was as follows: "The substance expectorated one and a half inches long, five-eighths of an inch wide, is cheese; it must have been lodged in some fossa or in a pouched osophagus formed by adhesion to a tubercular gland at the bifurcation of the trachea and after irritation from its presence or during an attack of coughing ejected." The patient disliked cheese; only twice during the year had she partaken of such an article of diet-namely, once during August and the last time on Dec. 26 th. The substance was ejected on Feb. 5th, so that it must have lain in the pouch for at least six weeks without giving rise to any symptoms.

The points of interest are the size of the piece of cheese and the length of time it was in the pouch. The question will also arise, What, if any, are the powers of digestion possessed by the glands lining the csophagus?

Brierley Hill. 